Dear Author,

Please, note that changes made to the HTML content will be added to the article before publication, but are not reflected in this PDF.

Note also that this file should not be used for submitting corrections. 


\section{AUTHOR QUERY FORM}

\begin{tabular}{|l|l|l|}
\hline & Journal: JALCOM & Please e-mail or fax your responses and any corrections to: \\
& & E-mail: corrections.esch@elsevier.sps.co.in \\
\hline ELSEVIER & Article Number: 32155 & Fax: +31204852799 \\
\hline
\end{tabular}

Dear Author,

Please check your proof carefully and mark all corrections at the appropriate place in the proof (e.g., by using on-screen annotation in the PDF file) or compile them in a separate list. Note: if you opt to annotate the file with software other than Adobe Reader then please also highlight the appropriate place in the PDF file. To ensure fast publication of your paper please return your corrections within 48 hours.

For correction or revision of any artwork, please consult http://www.elsevier.com/artworkinstructions.

Any queries or remarks that have arisen during the processing of your manuscript are listed below and highlighted by flags in the proof. Click on the ' $Q$ ' link to go to the location in the proof.

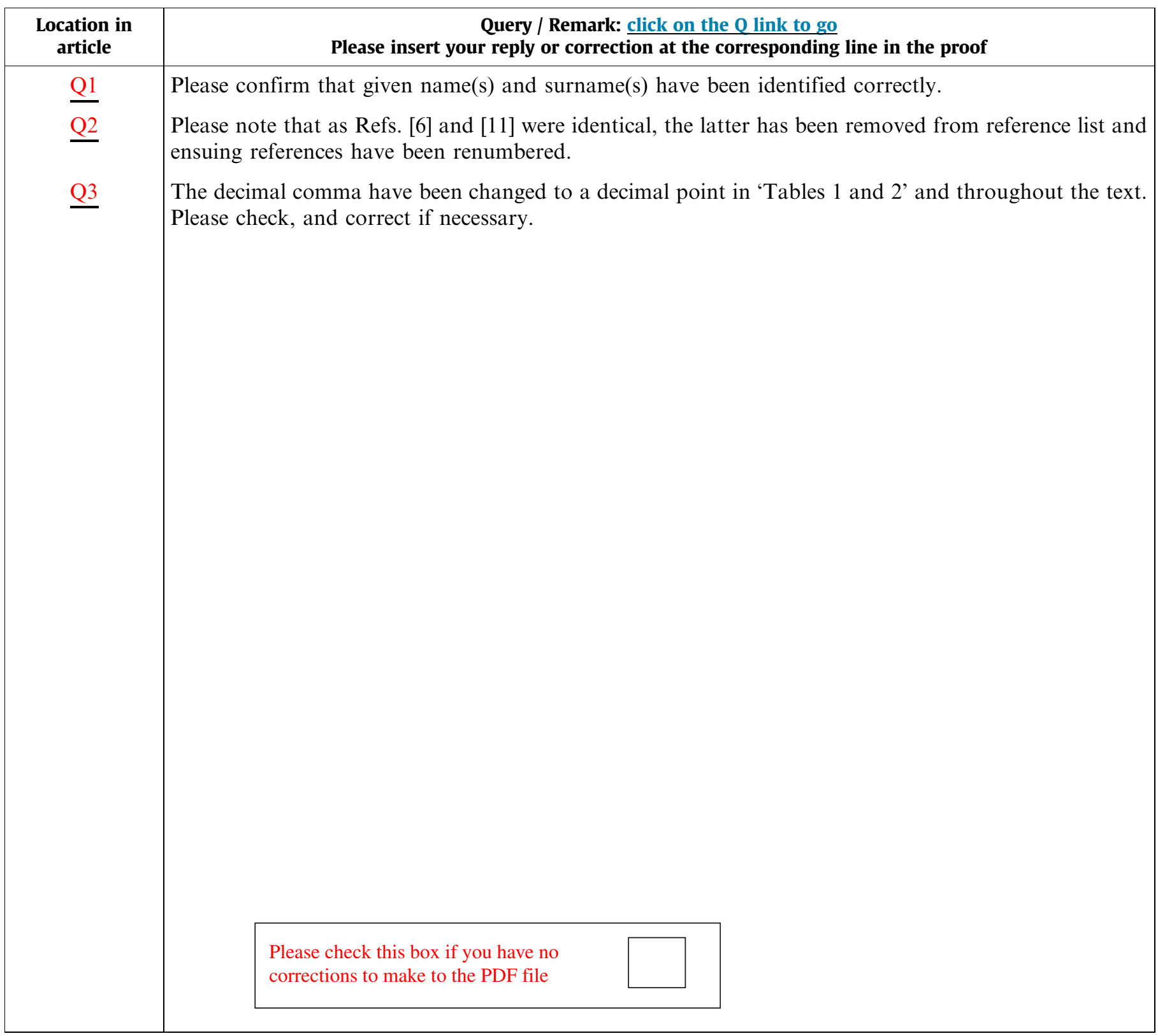

Thank you for your assistance. 


\section{Graphical abstract}

Influence of ferrite stabilizing elements and Co on structure and magnetic properties of carbon-encapsulated iron nanoparticles synthesized in thermal plasma jet

pp $x x x-x x x$

Z. Karoly, J. Szepvolgyi, W. Kaszuwara, O. Łabędź, M. Bystrzejewski*

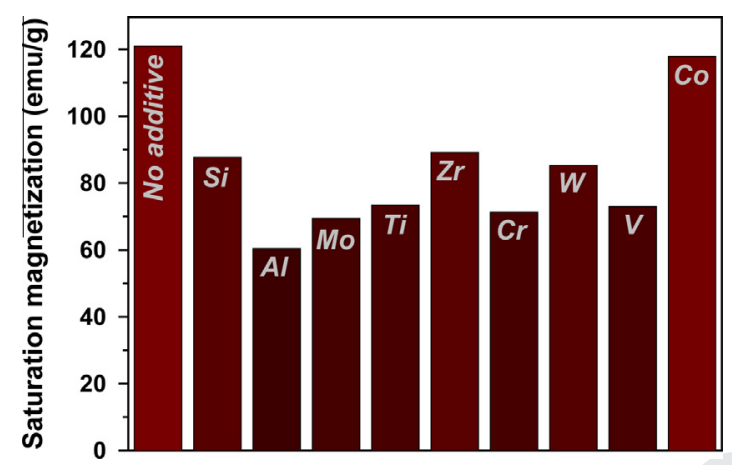

Highlights

- Inclusion of ferrite stabilizing elements reduces the diameter of CEINs. • Inclusion of ferrite stabilizing elements increases the amount of austenite. • Inclusion of $\mathrm{Al}, \mathrm{Ti}, \mathrm{Cr}$ and $\mathrm{V}$ causes formation of few layer graphene. • Magnetic performance of CEINs can be largely improved by post annealing. 


\title{
Influence of ferrite stabilizing elements and Co on structure and magnetic properties of carbon-encapsulated iron nanoparticles synthesized in thermal plasma jet
}

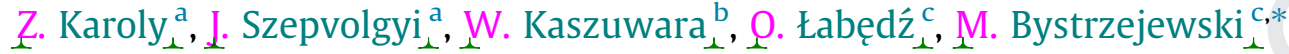 \\ a Chemical Research Centre, Hungarian Academy of Sciences, Pusztaszeri ut. 59-67, 1025 Budapest, Hungary \\ ${ }^{\mathrm{b}}$ Warsaw University of Technology, Faculty of Materials Science and Engineering, Wołoska 141 str., 02-507 Warsaw, Poland \\ ${ }^{c}$ University of Warsaw, Dept of Chemistry, Pasteur 1 str., 02-093 Warsaw, Poland
}

\section{A R T I C L E I N F O}

\section{Article history:}

Received 5 July 2014

Received in revised form 4 September 2014

Accepted 5 September 2014

Available online $\mathrm{xxxx}$

\section{Keywords:}

Magnetic materials

Magnetic properties

Phase composition

Austenite

Annealing

\begin{abstract}
A B S T R A C T
The encapsulation of Fe nanoparticles in protective carbon coatings always leads to formation of undesired paramagnetic austenite phase. Various ferrite stabilizing elements were included in the synthesis process to verify whether their inclusion may minimize the austenite content in carbon-encapsulated iron nanoparticles synthesized in thermal plasma jet. Eight ferrite stabilizing elements ( $\mathrm{Si}, \mathrm{Al}, \mathrm{Mo}, \mathrm{Ti}$, $\mathrm{Zr}, \mathrm{Cr}, \mathrm{W}$ and $\mathrm{V}$ ) and one austenite promoting additive (Co) were tested. Their influence on the synthesis yield, phase composition, morphology and magnetic properties of carbon-encapsulated iron nanoparticles was studied. It was found that the addition of ferrite stabilizers strongly influences the diameter distribution, graphitization degree, phase composition and magnetic properties. Contrary to the thermodynamic predictions the inclusion of ferrite stabilizing elements caused a substantial worsening of magnetic performance in carbon-encapsulated iron nanoparticles. It has been also shown that the subsequent heat treatment of carbon-encapsulated iron nanoparticles significantly improves their magnetic properties.
\end{abstract}

(c) 2014 Published by Elsevier B.V.

\section{Introduction}

Carbon-encapsulated magnetic nanoparticles, frequently named as "carbon encapsulates", are a core-shell type nanomaterial with a broad perspective of applications. Generally, the shell in these nanostructures is of great importance, because it effectively protects the core material from unwanted and uncontrollable processes, e.g. oxidation, corrosion and agglomeration [1]. Carbon encapsulates are considered as a unique platform which delivers a very original solution to preserve the inherent physical and chemical properties of bare metal nanoparticles. The carbon coating in carbon encapsulates is the best coating agent among other encapsulating materials (gold, polymers, boron nitride) because it is light, impermeable and has high stability in contact with various aggressive chemical reagents (non-oxidative mineral and organic acids, bases, greases, oils) [2]. Moreover, the carbon coating possesses high thermal stability because it does not undergo gasification under oxygen atmosphere at temperature below $400-450{ }^{\circ} \mathrm{C}[3]$.

\footnotetext{
* Corresponding author. Tel.: +48 228220211.

E-mail address: mibys@chem.uw.edu.pl (M. Bystrzejewski).
}

Carbon-encapsulated magnetic nanoparticles can be fabricated by a variety of synthesis routes. These approaches can be divided into (i) low temperature and (ii) high temperature routes. The first group primarily includes pyrolysis based processes and chemical vapor deposition [4-7]. The low temperature approaches do not require large energy input, however on the other hand have limited selectivity [7]. The high temperature routes (e.g. carbon arc discharge, thermal plasma, flame spray synthesis) consume more energy, however, are capable to fabricate carbon encapsulates in a continuous manner and with high selectivity [8-10,6]. Iron is the most frequent encapsulated element in carbon. This is because the best magnetic performance of Fe over other transition metals. Unfortunately, the encapsulation of Fe always leads to broad phase composition. The products contain bcc $\mathrm{Fe}, \mathrm{Fe}_{3} \mathrm{C}$ and $f c c \mathrm{Fe}-\mathrm{C}$ (austenite) nanoparticles encapsulated in carbon. The presence of austenite is highly undesirable because this phase is paramagnetic (at room temperature) and diminishes the overall magnetic moment. The data published in previous papers show that the relative amount of austenite in carbon-encapsulated iron nanoparticles (CEINs) can reach even $30 \%[11,12]$. The goal of this work is to verify whether the inclusion of ferrite stabilizing elements (FSE) in the synthesis process of carbon-encapsulated iron 
nanoparticles may minimize the formation of unwanted austenite phase. The thermodynamic predictions of the Fe-FSE system evidence that the presence of all studied elements hampers the formation of austenite and stabilize the ferrite phase. The phase diagrams of these Fe-FSE systems are shown in Supplementary Data. This work partially corresponds to the previous paper in which the influence of $\mathrm{Al}$ on magnetic properties of carbon-encapsulated iron nanoparticles synthesized via carbon arc discharge was studied [13].

\section{Experimental}

The synthesis of carbon-encapsulated iron nanoparticles was carried out using a flow-through radio frequency thermal plasma reactor. The reactor comprises of two sections, i.e. the plasma torch and the water-cooled chamber, in which the products undergo condensation. The experimental system was described in details elsewhere [14]. All tests were carried out under atmospheric pressure. The RF power (22$23 \mathrm{~kW}$ ) was supplied by a generator operating at 2-3 $\mathrm{MHz}$. Argon was used as a plasma gas $(15 \mathrm{slpm})$, while the sheath gas was $\operatorname{Ar}(40 \mathrm{slpm})$ mixed with He (20 slpm). The starting reactants (Fe powder, an additive and ethanol) were axially introduced via a water-cooled probe located at the center of the plasma torch. Ethanol (purity at least 98\%) was fed by a pneumatic feeder with a flow rate of $12.5 \pm 1.5 \mathrm{ml} / \mathrm{min}$. The Fe powder with the mean grain size of $6-9 \mu \mathrm{m}$ (as declared by the manufacturer) was used. Nine various additives ( $\mathrm{Si}, \mathrm{Al}, \mathrm{Mo}, \mathrm{Ti}, \mathrm{Zr}, \mathrm{Cr}, \mathrm{W}, \mathrm{V}$ and $\mathrm{Co}$ ) were in a form of fine powders (the mean grain size between $10 \mu \mathrm{m}$ and $50 \mu \mathrm{m}$ ). The metal powders (pure Fe or Fe-additive mixture (90-10 wt.\%)) were delivered to the torch by argon ( $5 \mathrm{slpm}$ ) with a feed rate of $1.6-5.8 \mathrm{~g} / \mathrm{min}$. The feed rate for each delivered mixture and the corresponding flow rate of the collected product are given in Table S1 (see Supplementary Data).

The as-synthesized (raw) products were collected from the reactor walls only. In each test some amount of the solid products were also present in the bottom of the reactor. These products consisted primarily of the non-processed starting metal particles, and therefore they were not collected. The raw products were subjected to purification in order to irreversibly remove the non-encapsulated metallic particles and these particles, which were encapsulated in permeable (defected) carbon coatings. The purification procedure included $24 \mathrm{~h}$ of boiling in $3 \mathrm{M} \mathrm{HCl}$ with subsequent washing with excess water and ethanol. The mass of the product recovered after purification was monitored. The chemical composition of the raw and purified products was evaluated by thermogravimetry under oxygen atmosphere (the full procedure and the corresponding curves are shown in Supplementary Data). The morphology of the raw and purified products was studied by transmission electron microscopy (TEM, Zeiss Libra 120 operated at $120 \mathrm{kV}$ ). The phase composition studies were conducted on a Bruker D8 diffractometer using a $\mathrm{Cu} \mathrm{K} \alpha$ radiation in a $2 \Theta$ range between 10 and 70 with a step of 0.02 deg. Raman spectra were acquired using a dispersive spectrometer (Jobin Ivon T-64000) equipped a $514.5 \mathrm{~nm}$ excitation laser. Magnetic measurements were carried out at $25^{\circ} \mathrm{C}$ using a vibrating magnetometer (Lake Shore 668 ). The measured magnetization was referred to the total mass of the studied sample.

\section{Results and discussion}

\subsection{Process efficiency}

Fig. 1a shows the product formation rate of carbon-encapsulated iron nanoparticles. The product formation rate is the defined as the ratio of the flow rate of the collected product from the reactor walls and the total feed rate of the starting reagents (i.e. ethanol, Fe and the additive). Generally, the product formation rate for most of the used additives is between $5 \%$ and $8 \%$. A substantially higher value is observed for the test conducted with the inclusion of $\mathrm{W}(11 \%)$ and $\mathrm{Zr}(18 \%)$. The higher values of the product formation rate plausibly result from the presence of corresponding carbides, which may interfere the product formation rate (i.e. WC and ZrC, see Section 3.3 for more details). Fig. 1b shows the purification yield for CEINs synthesized with the addition of various ferrite stabilizing additives. The purification yield is defined as the ratio of the starting and the recovered mass of the sample which was subjected to purification. In other words, the purification yield visualizes how much of the raw product is irreversibly dissolved during acid treatment. The purification yield varies in a relatively broad range, i.e. between $23 \%$ and $52 \%$. For the correct interpretation of these results one
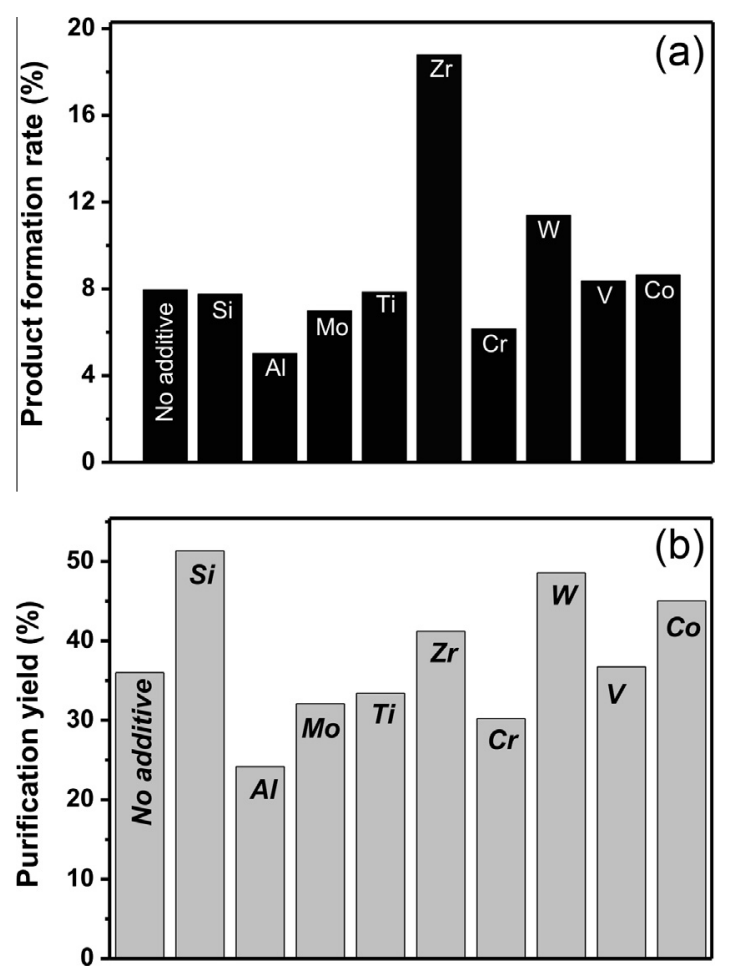

Fig. 1. Product formation rate (a) and purification yield (b) of carbon-encapsulated iron nanoparticles synthesized with addition of various ferrite stabilizing elements.

has to refer to chemical stability of the additives (and the corresponding carbides) in boiled $3 \mathrm{M} \mathrm{HCl}$. Iron, cobalt, aluminum, titanium and chromium are the metals that are readily soluble in hydrochloric acid. The other studied additives ( $\mathrm{W}, \mathrm{Zr}, \mathrm{V}$, Mo and $\mathrm{Si}$ ) are resistant to $\mathrm{HCl}$. Among the carbides, only $\mathrm{Al}_{4} \mathrm{C}_{3}$ and $\mathrm{CO}_{2} \mathrm{C}$ are the compounds that are easily leached by hydrochloric acid. The pattern in Fig. 2 shows that the products synthesized with the inclusion of $\mathrm{Si}, \mathrm{Zr}, \mathrm{W}$ and $\mathrm{Co}$ plausibly contain, in addition to CEINs, carbides or pure metal crystallites, which can appear in the encapsulated and non-encapsulated form.

The data from Fig. $1 \mathrm{a}$ and $\mathrm{b}$ along with the operational details from Table S1 can be used to estimate the overall process efficiency. The overall process efficiency (TPE) is a number, which determines the mass of the purified products, which is available in a unit of time (e.g. $g / h$ ). This parameter can be calculated in the following way: TPE $(g / h)=P F R \cdot P Y \cdot F R S(g / h)$, where TPE is the overall process efficiency, PFR is the product formation rate (dimensionless), PY is the purification yield (dimensionless) and FRS is the flow rate of the starting reagents (i.e. ethanol, iron and the additive). The TPE values are shown in Fig. S1. The pattern in Fig. S1 is generally similar to the diagrams presented in Figs. 1 and 2. The process efficiency varies between ca. $9 \mathrm{~g} / \mathrm{h}$ for $(\mathrm{Al})$ and ca. $62 \mathrm{~g} / \mathrm{h}(\mathrm{Zr})$. The data show that the inclusion of $\mathrm{W}$ and $\mathrm{Zr}$ results in a (at least) 1.5 -fold increase of the process efficiency.

\subsection{Morphology}

The representative TEM images of the products are shown in Fig. 2. Irrespectively of the applied additive the samples contain nanosized particles. The nanoparticles are covered by a thin carbon coating (a few $\mathrm{nm}$ in thickness). This observation directly proves that the encapsulation process was successful. In the case of the sample obtained from pure Fe (without any addition) most of the nanoparticles have the diameter in the range between $10 \mathrm{~nm}$ and $70 \mathrm{~nm}$ (Fig. 2a). The inclusion of ferrite stabilizing elements 

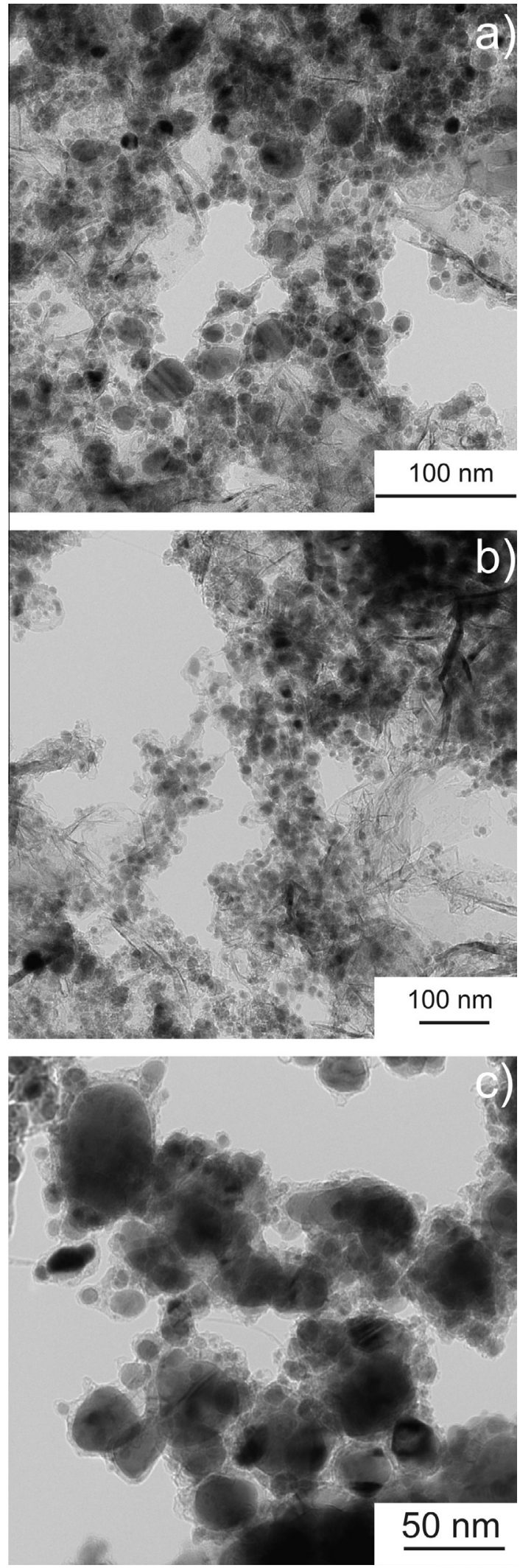

Fig. 2. Representative TEM images of carbon-encapsulated iron nanoparticles synthesized from pure $\mathrm{Fe}(\mathrm{a})$, with addition of $\mathrm{Al}(\mathrm{b})$ and $\mathrm{Zr}$ (c). decreases the diameter of CEINs. The nanoparticles have substantially smaller diameter, i.e. $10-30 \mathrm{~nm}$ for Al (Fig. 2b) and 20-50 $\mathrm{nm}$ for $\mathrm{Zr}$ (Fig. 2c). The same morphological features are observed in the products synthesized with the addition of other
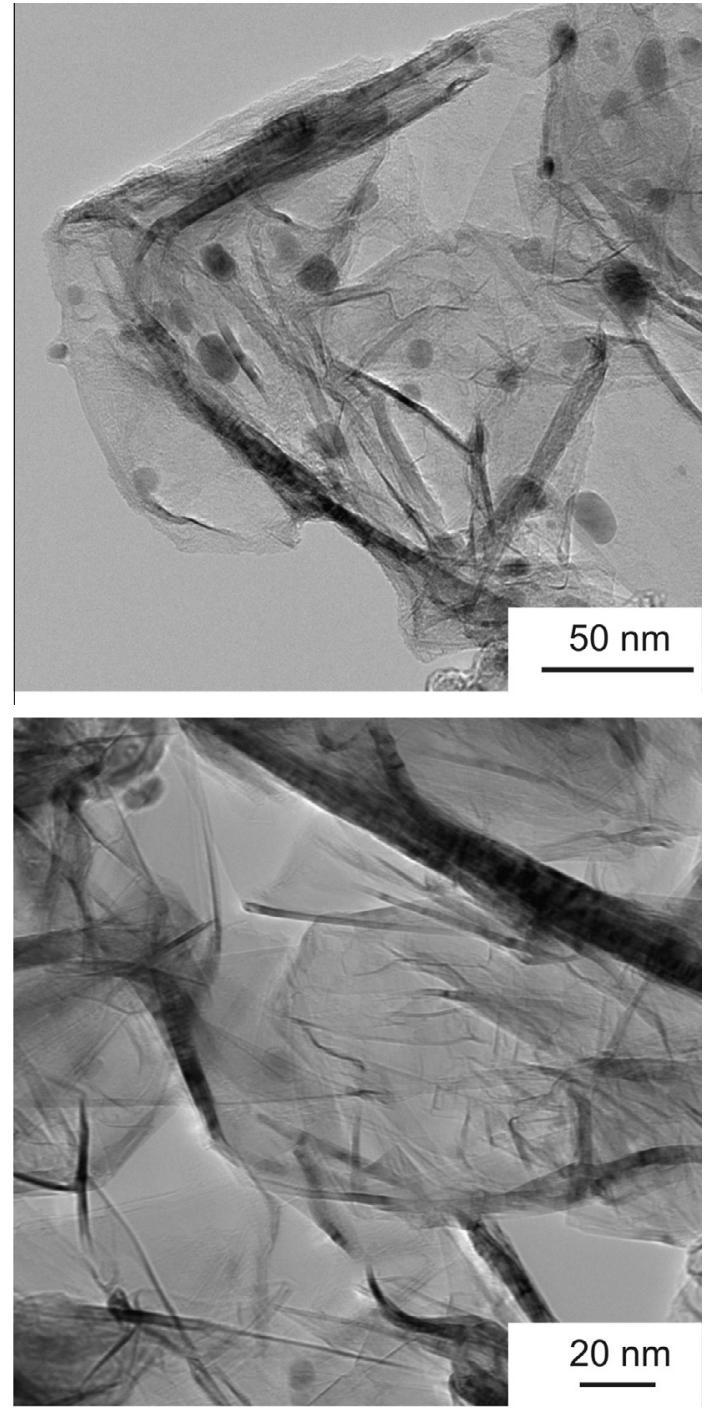

Fig. 3. Representative TEM images of a few-layer graphene from product synthesized with addition of $\mathrm{Al}$.

ferrite stabilizing elements. The carbon coatings are easily observed and their thickness is up to $3-5 \mathrm{~nm}$. This thickness corresponds up to 10-15 stacked curved graphene layers. Importantly, in some cases the products also contain few layer graphene (selected images are shown in Fig. 3). The graphene structures have the thickness between $5 \mathrm{~nm}$ and $20 \mathrm{~nm}$ (the thickness was evaluated from the size of the bending zones, which are clearly visible on the images). The few layer graphene sheets was observed in the products synthesized from pure Fe and with the addition of: $\mathrm{Al}$, $\mathrm{Ti}, \mathrm{Cr}$ and $\mathrm{V}$. These structures were observed very occasionally in the samples obtained from $\mathrm{Fe}-\mathrm{Mo}, \mathrm{Fe}-\mathrm{Co}$ and $\mathrm{Fe}-\mathrm{Si}$. The presence of few-layer graphene was not found in the product obtained with the inclusion of $\mathrm{Zr}$ and $\mathrm{W}$. This observation shows that some ferrite stabilizing elements also influences the process selectivity.

A rough analysis of TEM images demonstrates that the inclusion of various ferrite stabilizing additives affects the diameter distribution of carbon-encapsulated iron nanoparticles. Therefore, the statistical analysis (with the corresponding histograms) was performed. At least 150 objects on the microscopic images were analyzed and the mean diameter was evaluated from the log-normal fit. Fig. 4a and b show the selected histograms of diameter distribution for carbon-encapsulated iron nanoparticles synthesized with the addition of $\mathrm{Si}$ and $\mathrm{Al}$, respectively (the histograms for 

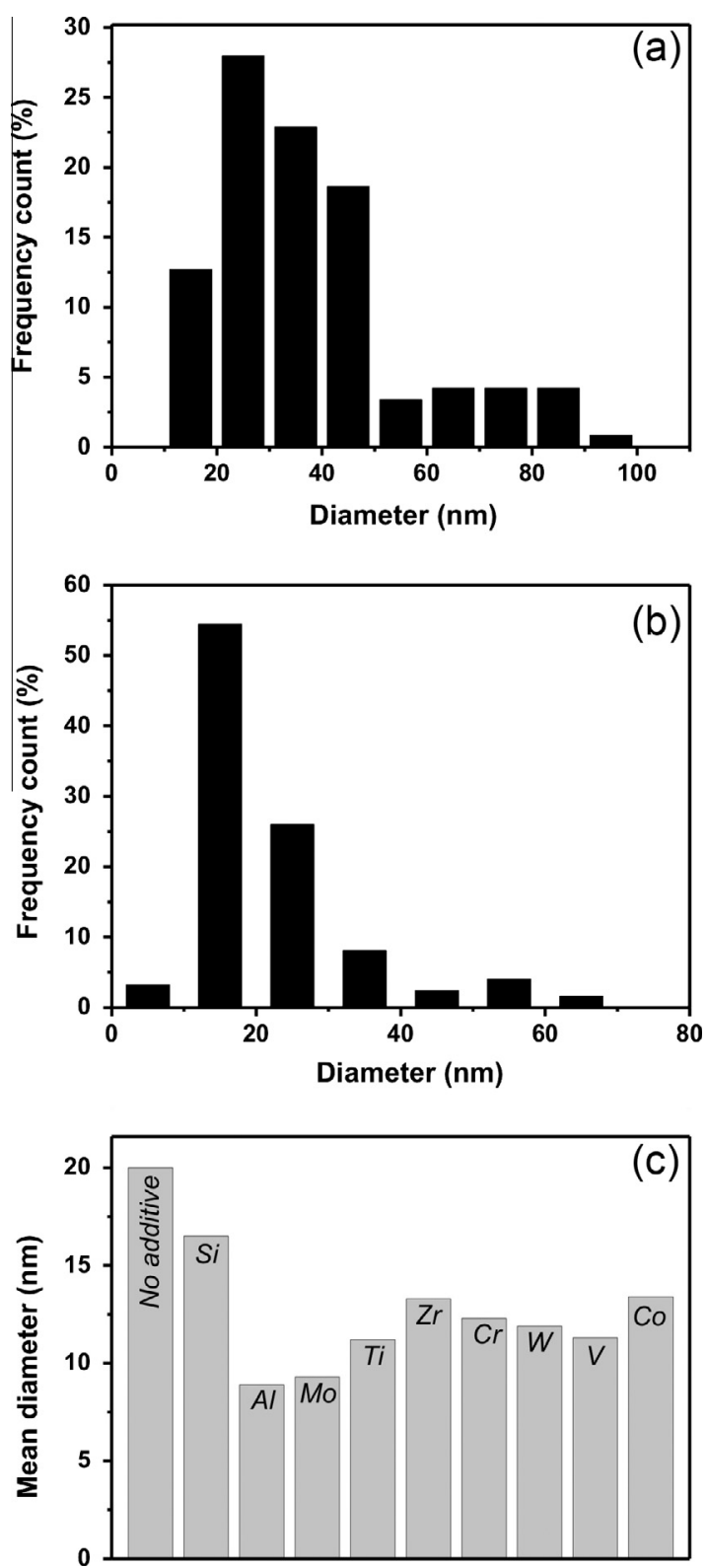

Fig. 4. Selected diameter distributions of CEINs synthesized with addition of Si (a), $\mathrm{Al}$ (b) and mean diameter for various ferrite stabilizing elements (c).

other samples are shown in Fig. S2). The mean diameter of CEINs synthesized with the addition of various ferrite stabilizing elements is shown in Fig. 4c. The mean diameter of carbon encapsulates synthesized in the presence of FSE is reduced in comparison to CEINs obtained from pure Fe. The largest reduction (more than twofold) is observed for $\mathrm{Al}$ and Mo. In the case of other additives ( $\mathrm{Ti}, \mathrm{Zr}, \mathrm{Cr}, \mathrm{W}, \mathrm{V}$ and $\mathrm{Co}$ ) the mean diameter is between $10 \mathrm{~nm}$ and $13 \mathrm{~nm}$. Interestingly, the reduction of the mean diameter in the case of $\mathrm{Si}$ is the lowest (i.e. from $20 \mathrm{~nm}$ to $17 \mathrm{~nm}$ ). The recent studies show that the diameter distribution of CEINs synthesized in thermal plasma jet depends primarily on the sublimation kinetics of pristine Fe grains [15]. The larger grains, due to their greater weight, sublimate slower because their residence time in the high temperature zone of the plasma flame is limited. The slower sublimation corresponds to the lower density of Fe vapor and consequently leads to fewer collision/nucleation frequency, which finally results in particles of smaller diameter. However, these considerations are not adequate to explain the pattern in Fig. 4c, because the starting Fe powder and the additives have a comparable grain size. Thus, the observed variation in the mean diameter is plausibly related with the presence of various additives. This finding fairly agrees with the other literature data, which shows that the studied additives influence the grain size of carbon steel. One has to be aware that the direct analogy between carbon steel and carbon-encapsulated iron nanoparticles is only an a very rough assumption. Silicon, titanium, tungsten, vanadium, zirconium, aluminum and molybdenum are the elements, which generally promote the fine grain structure in carbon steel (their presence limits or refines the grains size) [16]. On the other hand, the presence of chromium and cobalt presumably results in the grain coarsening [17]. The comparison of these literature data with the pattern in Fig. 4c partially explains the role of the additive in controlling the diameter distribution. The compatibility is found for most of the additives, however, except of Co and $\mathrm{Cr}$.

\subsection{Structure and chemical composition}

Fig. 5 shows the selected X-ray diffraction patterns of the purified products (the patterns for other raw and purified samples are shown in Figs. S3 and S4). The qualitative phase composition of all products is listed in Table 1. The sample synthesized from pure $\mathrm{Fe}$ is comprised of four crystalline phases: graphitic carbon, $b c c \mathrm{Fe}, f c c$ $\mathrm{Fe}-\mathrm{C}$ (austenite) and iron carbide. The (002) reflection for graphitic carbon (located at $26 \mathrm{deg}$ ) is broadened. This is a consequence of its low thickness (3-5 nm, see TEM results) and topological defects (pentagons and heptagons) which are needed to introduce the curvature in graphene layers. The austenite phase is thermodynamically unstable at room temperature and should not be present. It is not an unexpected result since this phase was observed previously in CEINs by other researchers [11]. The encapsulation of austenite nanoparticles in carbon blocks the spontaneous transition of fcc Fe-C to bcc Fe and $\mathrm{Fe}_{3} \mathrm{C}$. The inclusion of ferrite stabilizing changes the phase composition (Table 1). First of all, the (002) reflection from graphite is absent or has very weak intensity. This is due to the fact that this phase is of much lower crystallinity than other phases present in the analyzed samples. The raw products have generally simpler phase composition in comparison to the purified materials. The $(110)$ reflection from bcc Fe is the strongest feature in all samples. The bare metallic phases and/or their respective carbides are present in the products obtained with the inclusion of the additives, however, the corresponding reflections are of relatively weak intensity. In the case of purified CEINs the fcc $\mathrm{Fe}-\mathrm{C}$ phase is observed in the products obtained from pure Fe and with the addition of the following additives: Mo, Ti, Zr, Cr, W and V. These findings demonstrate, contrary to the thermodynamic predictions, that the inclusion of ferrite stabilizing elements does not eliminate the unwanted austenite phase. Moreover, most of FSE (Mo, Ti, $\mathrm{Zr}, \mathrm{Cr}, \mathrm{W}$ and V) leads to the formation of the respective carbides. None of the reflections from the carbide phases were observed in the products obtained with the addition of $\mathrm{Si}, \mathrm{Al}$ and Co. Moreover, in the case of $\mathrm{Si}, \mathrm{Al}$, $\mathrm{Ti}, \mathrm{Zr}$ and $\mathrm{W}$ the XRD patterns show the presence of the bare metal crystallites. Since all of the carbides originating from the ferrite stabilizing additives are resistant to hydrochloric acid it cannot be unambiguously stated that the nanoparticles made of these carbides are encapsulated or not encapsulated in carbon. The same finding is found for $\mathrm{Si}, \mathrm{Zr}$ and $\mathrm{W}$ (these metals are also insoluble in $\mathrm{HCl}$ ). However, the detailed analysis of TEM images does not demonstrate the presence of other objects than carbon encapsulates and few layer graphene. This finding strongly suggest that the phases based on FSE additives are encapsulated in carbon. In fact, the literature review shows a few examples of carbon-encapsulated Si [18], W [19] and Zr [20] nanoparticles. Additionally, the (110) Fe reflection in all purified CEINs is broadened and spans the 

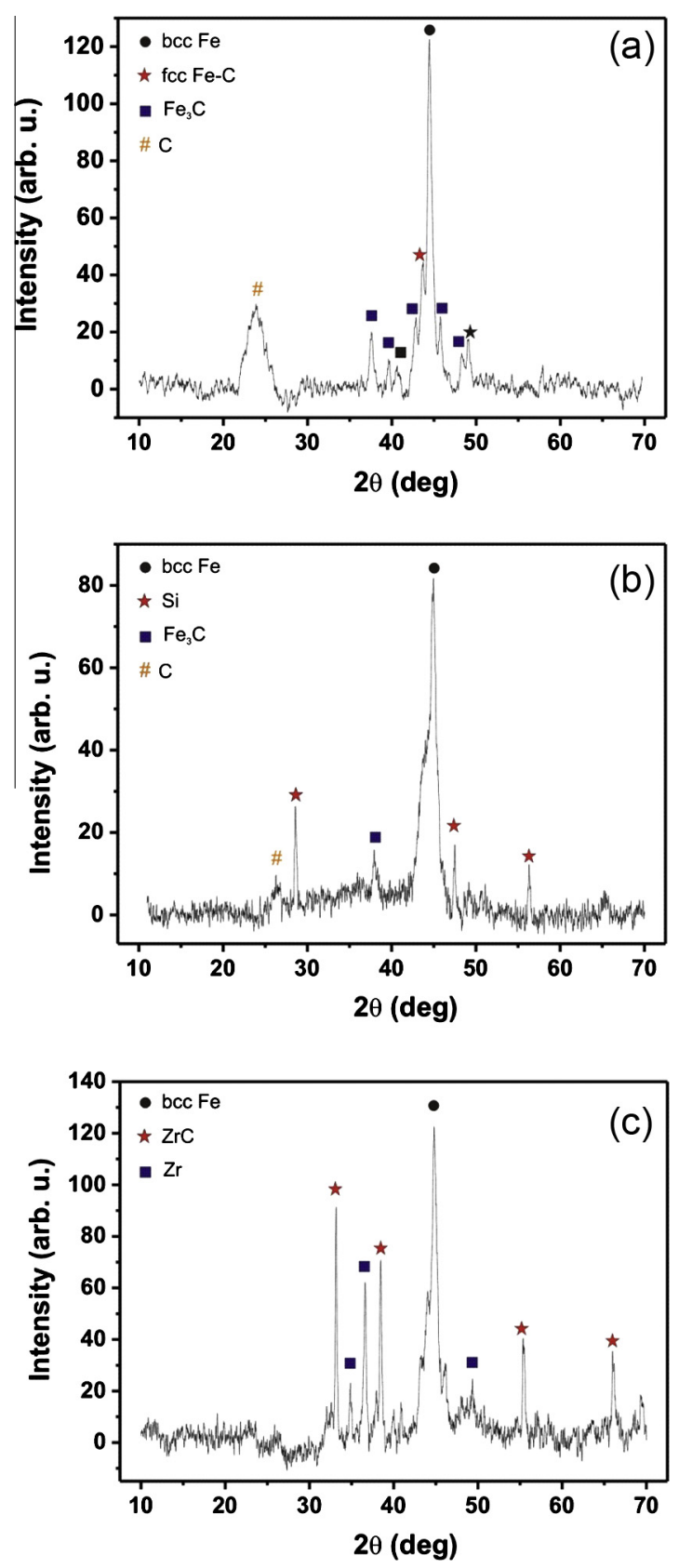

Fig. 5. Selectred powder XRD patterns of purified carbon-encapsulated iron nanoparticles synthesized from pure Fe (a), with addition of $\mathrm{Al}$ (b) and $\mathrm{Zr}$ (c).

Table 1

Q3 Phase composition and G/D ratio values of carbon-encapsulated iron nanoparticles synthesized with the addition of various ferrite stabilizing additives.

\begin{tabular}{llll}
\hline Additive & $\begin{array}{l}\text { Phases identified } \\
\text { in raw CEINs }\end{array}$ & $\begin{array}{l}\text { Phases identified } \\
\text { in purified CEINs }\end{array}$ & $G / D$ \\
\hline Pure Fe & bcc Fe, fcc Fe-C, $\mathrm{Fe}_{3} \mathrm{C}$ & $\mathrm{C}$, bcc Fe, fcc Fe-C, Fe ${ }_{3} \mathrm{C}$ & 1.80 \\
$\mathrm{Si}$ & bcc Fe, fcc Fe-C, $\mathrm{Si}$ & $\mathrm{C}$, bcc Fe, $\mathrm{Si}, \mathrm{Fe}_{3} \mathrm{C}$ & 0.94 \\
$\mathrm{Al}$ & bcc Fe, $\mathrm{Al}$ & $\mathrm{C}$, bcc Fe, $\mathrm{Al}$ & 1.76 \\
$\mathrm{Mo}$ & bcc Fe, $\mathrm{Mo}_{2} \mathrm{C}$ & bcc Fe, fcc Fe-C, $\mathrm{Mo}_{2} \mathrm{C}$ & 1.29 \\
$\mathrm{Ti}$ & bcc Fe, TiC & bcc Fe, fcc Fe-C, Ti, TiC & 1.96 \\
$\mathrm{Zr}$ & bcc Fe, fcc Fe-C, $\mathrm{ZrC}$ & bcc Fe, fcc Fe-C, $\mathrm{Zr}, \mathrm{ZrC}$ & 1.13 \\
$\mathrm{Cr}$ & bcc Fe & bcc Fe, fcc Fe-C Cr $\mathrm{C}_{3}$ & 1.62 \\
$\mathrm{~W}$ & bcc Fe, W, WC & bcc Fe, fcc Fe-C, W, WC & 1.26 \\
$\mathrm{~V}$ & bcc Fe, $\mathrm{V}_{2} \mathrm{C}$ & bcc Fe, fcc Fe-C, Fe ${ }_{3} \mathrm{C}, \mathrm{C}, \mathrm{V}_{2} \mathrm{C}, \mathrm{VC}$ & 1.78 \\
$\mathrm{Co}$ & bcc Fe-Co & bcc Fe-Co & 1.23 \\
\hline
\end{tabular}

2 theta range between ca. 40 and $45 \mathrm{deg}$. This range is typical for the strongest reflections of $\mathrm{Fe}_{3} \mathrm{C}$ phase. Therefore the presence of iron carbide crystallites cannot be excluded as well.

Further structural details were derived from Raman spectra. Raman spectroscopy is a powerful and sensitive technique which is frequently used to investigate the structural ordering in carbon materials. The first order Raman spectrum has two typical bands, i.e. the so-called $\mathrm{G}$ and $\mathrm{D}$ bands. The first spectral feature which is located at ca. $1585 \mathrm{~cm}^{-1}$ is related with in-plane vibrations of $\mathrm{C}_{-} \mathrm{C}$ bonds within the hexagonal lattice of graphene layers [21]. This band is associated with structural ordering and gets more intense in highly graphitized carbon materials. The D band, which is located at ca. $1340 \mathrm{~cm}^{-1}$, corresponds to structural (e.g. vacancies, heteroatoms) and topological (e.g. pentagons, heptagons) defects. The intensity of this feature is increased in carbon materials having low structural ordering. The so-called $G / D$ ratio is a common indicator of graphitization degree in carbon-based materials [22]. Please note, that this ratio should be evaluated not from the peak intensities. The proper way involves the deconvolution procedure and calculation the areas under the $G$ and $D$ bands. The selected Raman spectra are shown in Fig. 6. In the case of the product obtained with the addition of Si the spectrum contains a relatively weak band located at $519 \mathrm{~cm}^{-1}$. This feature corresponds to the lattice vibrations of $\mathrm{Si}$. In fact, the presence of silicon was also confirmed by X-ray diffraction studies. The evaluated values of $G / D$ ratio are listed in Table 1 . This parameter varies in a relatively narrow range, i.e. between 0.94 and 1.80 . This range corresponds to carbon materials with a moderate graphitization degree [23]. A rough look on the data in Table 1 does not bring any correlation between the additive and the $G / D$ ratio. All of the additives studied in this work are known as efficient catalyst of graphitization [24]. Nevertheless, none of them caused a substantial improvement of the graphitization degree. This finding might be due to the fact that the formation of carbon coating in CEINs is a rapid process, whilst the available literature data [24] concern the processes, which undergoes under low heating rates. Another interesting finding is the nearly linear correlation between the $G / D$ ratio and the purification yield (Fig. 7). As mentioned in the above sections the higher purification yield can result from the presence of carbides which are non-soluble in $\mathrm{HCl}$. The "tightness" of the carbon coating is the other factor (probably the most important one) which influences the encapsulation yield. The tight coating provides the essential barrier against the corrosion agent and provides the acid resistance to the encapsulated particles. The carbon coating is built of curved graphene layers and mimics the shape of the encapsulated nanoparticle. The topological defects are necessary to introduce the sufficient curvature and make the coating hermetic and impermeable to hydrochloric acid. Obviously the higher density of topological defects should improve the tightness of the carbon coating and simultaneously decrease the $G / D$ ratio. This statement explains the observed pattern in Fig. 7.

Table 2 shows the chemical composition of the raw and purified products. The chemical composition was evaluated from thermogravimetric curves (for details see corresponding comments in Supplementary Data). In the case of the raw products $\mathrm{Fe}$ is the main constituent and its content is between 69.5 and $89.9 \mathrm{wt} . \%$. The purification procedure results in a decrease of both the Fe and FSE content. This reduction is an effect of the elimination of nanoparticles by hydrochloric acid treatment. Importantly the total content of $\mathrm{Fe}$ and the additive is lower than the Fe content in the purified CEINs synthesized with pure Fe. The largest reduction is found in the test with $\mathrm{Al}$, in which the total content is only $46.4 \mathrm{wt} . \%$ (this value is 1.7 -fold lower in comparison to the test with pure Fe). In fact, this observation is in agreement with the purification yield, which is $36 \%$ and $24 \%$ for pure Fe and Al additive, respectively. Among all studied additives, the use of Co resulted in 

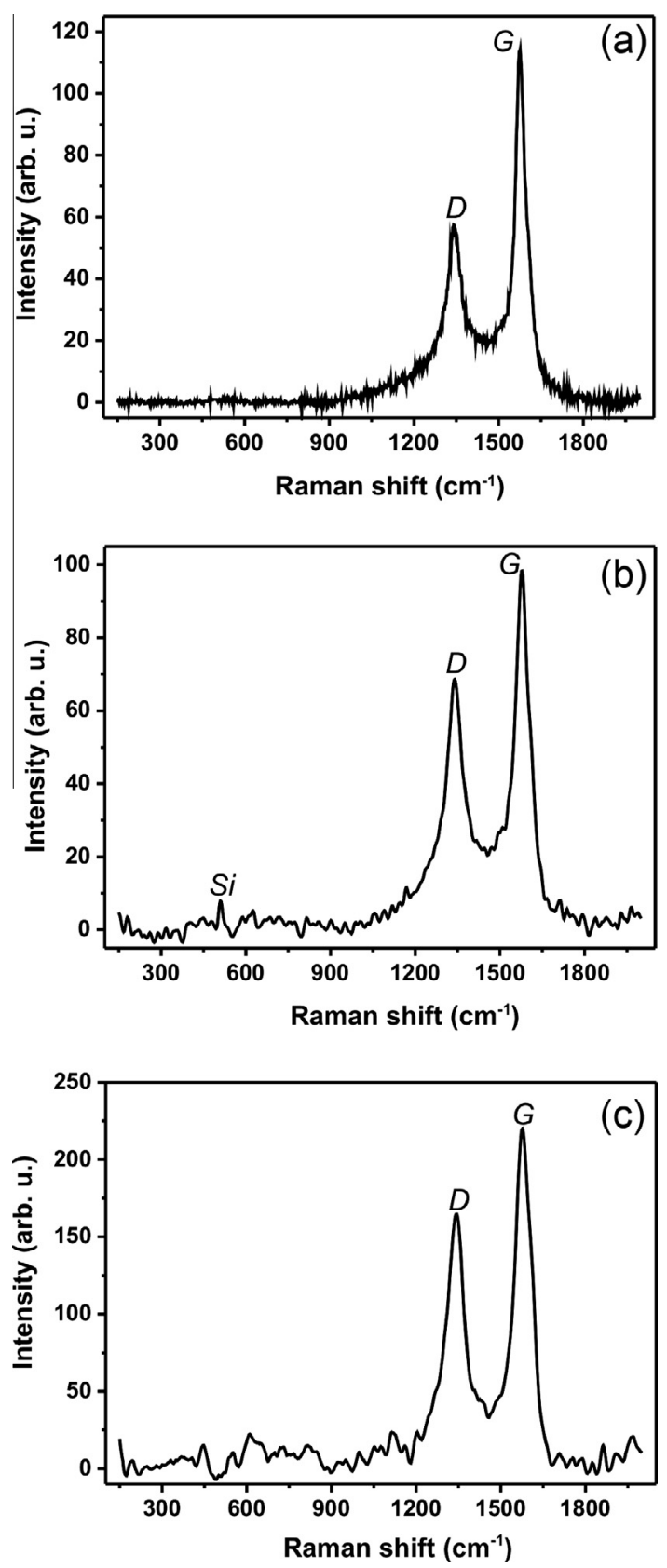

Fig. 6. Selected Raman spectra of purified of purified carbon-encapsulated iron nanoparticles synthesized from pure $\mathrm{Fe}(\mathrm{a})$, with addition of $\mathrm{Al}$ (b) and $\mathrm{Zr}$ (c).

a marginal reduction of the total metal content from 80.0 to 74.4 wt.\%.

The data from Table 2 clearly demonstrate that the inclusion of any ferrite stabilizing element causes the reduction of Fe content in the purified carbon-encapsulated iron nanoparticles. This observation can be explained using the phase composition results. As it follows from Table 2 the inclusion of FSE evokes (with an exception of $\mathrm{Si}$ and $\mathrm{Al}$ ) the formation of respective carbides. Therefore the available amount of carbon is diminished and it consequently leads to thinner or less-developed carbon coatings.

\subsection{Magnetic properties}

Fig. 8 shows the selected hysteresis loops for the raw and purified carbon-encapsulated iron nanoparticles. Both raw and purified products have typical soft ferromagnetic characteristics. The

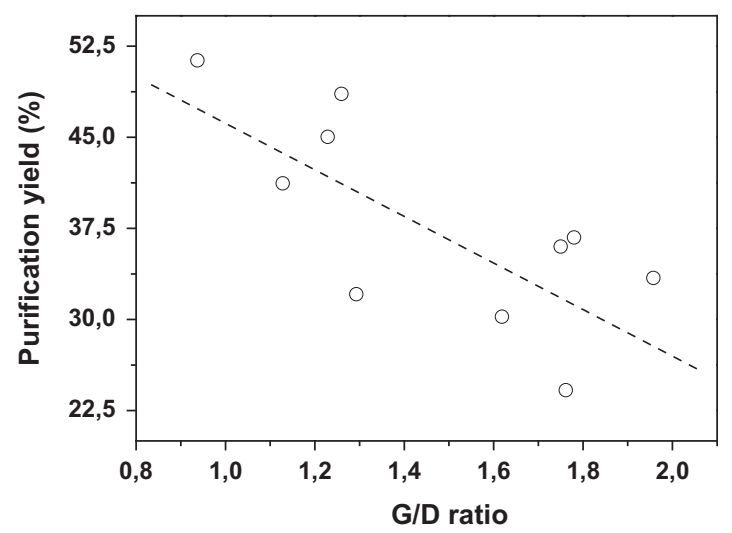

Fig. 7. Relation between purification yield and $G / D$ ratio.

saturation magnetization $\left(M_{s}\right)$ and coercive field values for all CEINs are listed in Table 3. The raw products have larger saturation magnetization in comparison to the purified samples. This is a consequence of different chemical composition, namely the Fe content in raw CEINs is higher in comparison to the purified products. The remnant magnetization for the raw and purified products is 1.5-6.3\% and 2.7-6.1\%, respectively. Both the raw and purified CEINs have a relatively low coercive field, which varies in a narrow range, i.e. between 40 and 173 Oe. The observed magnetic characteristics does not diverge from the magnetic performance of carbon-encapsulated iron nanoparticles fabricated using other techniques (carbon arc discharge [9], chemical vapor deposition [4], combustion synthesis [25], flame synthesis [10] and hydrothermal synthesis [26]).

Fig. 9a shows the values of saturation magnetization for the products obtained with all studied ferrite stabilizing elements. It is evident that all FSE additives worsens the magnetic performance. The highest reduction of $M_{s}$ is found for $\mathrm{Al}$ and Mo and in this case the saturation magnetization is lowered even twofold. Interestingly the inclusion of cobalt, which should promote and stabilize the paramagnetic austenite phase, virtually does not change the $M_{s}$ value. This picture is in total disagreement with the thermodynamic predictions and suggests that the growth of carbon-encapsulated iron nanoparticles undergoes under non-equilibrium conditions.

The observed saturation magnetization exclusively originates from the phases which are ferromagnetic, i.e. bcc-Fe and $\mathrm{Fe}_{3} \mathrm{C}$. The contribution from fcc $\mathrm{Fe}-\mathrm{C}$ can be neglected because the austenite phase is paramagnetic and its magnetic susceptibility is at least 4-5 orders of magnitude lower in comparison to the mentioned ferromagnetic constituents. The same scenario refers to carbides which were identified in phase composition studies (please note that all of these carbides have paramagnetic behavior). Obviously, the presence of either the FSE based carbides and/or fcc Fe-C will diminish the saturation magnetization. The pattern in Fig. 9a shows that the inclusion of FSE lowers the saturation magnetization by the factor 1.3-2.0. This reduction of $M_{s}$ cannot be exclusively caused by the presence of FSE based carbides. In the case, when all amount of the ferrite stabilizing agent added would be in a form a carbide then the saturation magnetization should be reduced by the factor not larger than 1.1 (the content of FSE in the initial Fe-FSE mixture is $10 \mathrm{wt} . \%$ ). Therefore it is very likely that the inclusion of ferrite stabilizing elements promotes the formation of austenite phase. The amount of austenite cannot be directly evaluated from the X-ray diffraction patterns, because the reflections from austenite and bcc Fe are overlapped. Nevertheless, it is possible to predict the relative content of austenite in a semi-quantitative way from the normalized values of saturation 
Table 2

Chemical composition of carbon-encapsulated iron nanoparticles synthesized with addition of various ferrite stabilizing additives.

\begin{tabular}{|c|c|c|c|c|c|c|}
\hline \multirow[t]{2}{*}{ Additive } & \multicolumn{3}{|c|}{ Raw CEINs } & \multicolumn{3}{|c|}{ Purified CEINs } \\
\hline & C (wt.\%) & $\mathrm{Fe}(\mathrm{wt} . \%)$ & Additive (wt.\%) & C (wt.\%) & $\mathrm{Fe}(\mathrm{wt} . \%)$ & Additive (wt.\%) \\
\hline Pure Fe & 11.1 & 89.9 & N.A. & 19.9 & 80.0 & N.A. \\
\hline $\mathrm{Si}$ & 22.7 & 69.5 & 7.8 & 35.6 & 57.9 & 6.5 \\
\hline $\mathrm{Al}$ & 19.6 & 72.3 & 8.1 & 53.6 & 41.7 & 4.7 \\
\hline Mo & 13.7 & 77.6 & 8.7 & 46.9 & 47.8 & 5.3 \\
\hline $\mathrm{Ti}$ & 10.6 & 80.4 & 9.0 & 33.9 & 59.5 & 6.6 \\
\hline $\mathrm{Zr}$ & 11.0 & 80.1 & 8.9 & 23.7 & 68.7 & 7.6 \\
\hline $\mathrm{Cr}$ & 12.5 & 78.7 & 8.8 & 34.9 & 58.6 & 6.5 \\
\hline W & 13.7 & 77.7 & 8.6 & 25.7 & 68.4 & 5.9 \\
\hline $\mathrm{V}$ & 10.1 & 81.2 & 8.7 & 29.0 & 64.1 & 6.9 \\
\hline Co & 11.3 & 79.9 & 8.8 & 25.6 & 67.0 & 7.4 \\
\hline
\end{tabular}

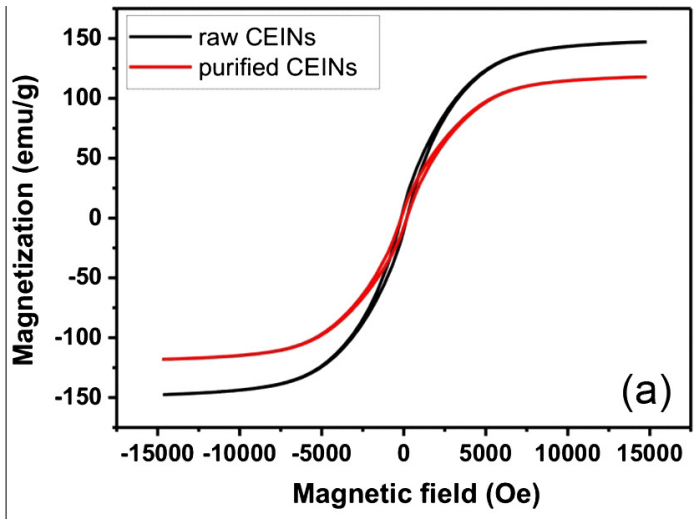

Table 3

Coercive force $\left(H_{c}\right)$ and saturation magnetization $\left(M_{s}\right)$ of carbon-encapsulated iron nanoparticles synthesized with addition of various ferrite stabilizing additives.

\begin{tabular}{llllll}
\hline \multirow{2}{*}{ Additive } & \multicolumn{2}{l}{ Raw CEINs } & & \multicolumn{2}{c}{ Purified CEINs } \\
\cline { 2 - 3 } \cline { 5 - 6 } & $H_{c}(\mathrm{Oe})$ & $M_{s}(\mathrm{emu} / \mathrm{g})$ & & $H_{c}(\mathrm{Oe})$ & $M_{s}(\mathrm{emu} / \mathrm{g})$ \\
\hline Pure Fe & 160 & 148 & & 146 & 120 \\
$\mathrm{Si}$ & 136 & 126 & & 123 & 88 \\
$\mathrm{Al}$ & 40 & 138 & & 52 & 61 \\
$\mathrm{Mo}$ & 66 & 141 & & 81 & 70 \\
$\mathrm{Ti}$ & 68 & 139 & & 89 & 74 \\
$\mathrm{Zr}$ & 163 & 125 & & 110 & 89 \\
$\mathrm{Cr}$ & 54 & 127 & & 57 & 71 \\
$\mathrm{~W}$ & 117 & 116 & & 121 & 85 \\
$\mathrm{~V}$ & 74 & 132 & & 99 & 73 \\
Co & 173 & 147 & 171 & 118 \\
\hline
\end{tabular}
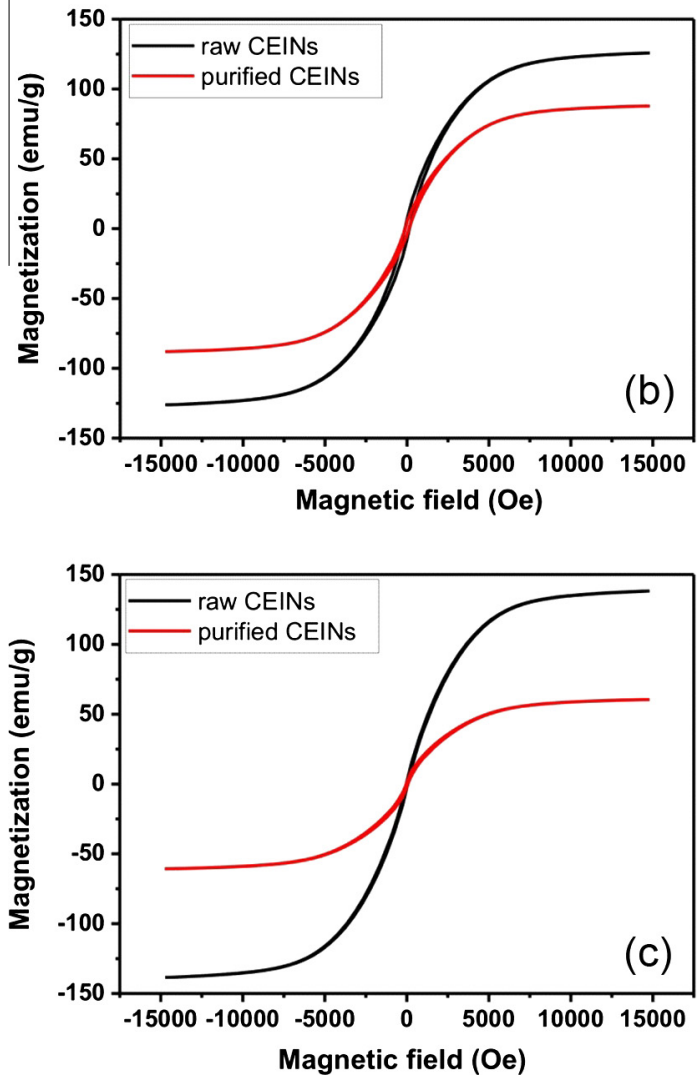

Fig. 8. Selected magnetic hysteresis loops for raw and purified CEINs obtained from pure $\mathrm{Fe}(\mathrm{a})$, with addition of $\mathrm{Al}(\mathrm{b})$ and $\mathrm{Zr}(\mathrm{c})$

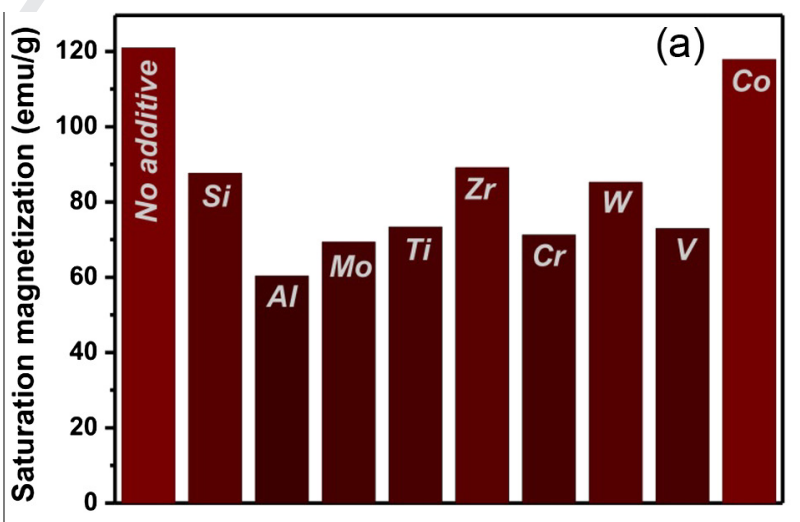

magnetization. The normalization procedure includes dividing the saturation magnetization by the total Fe content. Please note that the total Fe content is a value which visualizes the iron which is

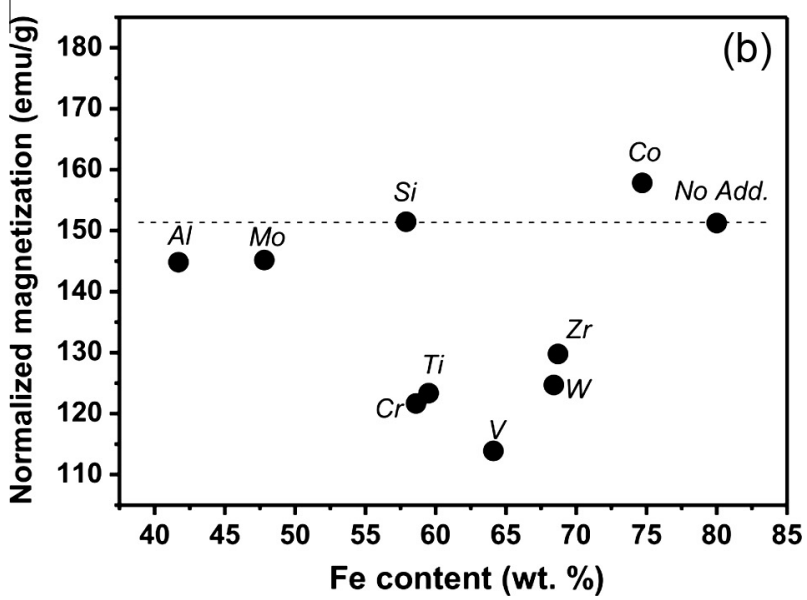

Fig. 9. Saturation magnetization (a) and normalized saturation magnetization (b) of purified CEINs synthesized with addition of various ferrite stabilizing elements. 
present in all Fe-bearing phases that are present in CEINs, i.e. bcc $\mathrm{Fe}, \mathrm{fcc} \mathrm{Fe}-\mathrm{C}$ and $\mathrm{Fe}_{3} \mathrm{C}$. The normalized saturation magnetization vs. total Fe content for all studied ferrite stabilizing elements is shown in Fig. 9b. The normalized $M_{s}$ of ca. 145-155 emu/g is found for CEINs synthesized from pure $\mathrm{Fe}$ and with addition of $\mathrm{Al}, \mathrm{Si}$, Mo and $\mathrm{Co}$. This finding clearly proves that the relative content of both ferromagnetic phases (i.e. bcc $\mathrm{Fe}$ and $\mathrm{Fe}_{3} \mathrm{C}$ ) is on a comparable level in these products. As for example the normalized $M_{s}$ of $150 \mathrm{emu} / \mathrm{g}$ may correspond to the situation in which the relative mass fractions of bcc Fe and $\mathrm{Fe}_{3} \mathrm{C}$ are ca. 38 and 40 wt.\% (0.38* $220 \mathrm{emu} /$ $\mathrm{g}+0.40 * 165 \mathrm{emu} / \mathrm{g}=151 \mathrm{emu} / \mathrm{g})$. In the case of $\mathrm{Cr}, \mathrm{Ti}, \mathrm{Zr}, \mathrm{W}$ and $\mathrm{V}$ the normalized saturation magnetization is substantially lower, i.e. it decreases even to $113 \mathrm{emu} / \mathrm{g}$. Hence, a such reduction must be accompanied by an increased amount of the paramagnetic austenite phase.

\subsection{Improving the saturation magnetization by post annealing}

As it was shown above the inclusion of ferrite stabilizing elements worsens the magnetic performance of carbon-encapsulated iron nanoparticles. The magnetic studies showed that the FSE additives promote the formation of austenite phase and decrease the specific magnetization. As it follows from the literature on heat treating of steel, the hardened steel contains retained austenite and martensite phases. These phases can be transformed to a mixture of ferrite and iron carbide phases during tempering process. The tempering process includes slow heating to the desired temperature and subsequent slow cooling. The recent studies have shown that the optimal temperature at which the retained austenite decomposes is above $400{ }^{\circ} \mathrm{C}$ [27]. We have adopted this procedure to the purified CEINs obtained with the addition of Ti to verify whether the heat treatment can promote the decomposition of austenite and increase the magnetic performance. This material was chosen because it has substantially low saturation magnetization in comparison to the reference sample (i.e. obtained from pure Fe). The process was tracked by differential scanning callorimetry (DSC). Fig. S6 shows the DSC heating-cooling curve, which was acquired with the heating/cooling rate of $10^{\circ} \mathrm{C} / \mathrm{min}$ under nitrogen atmosphere. The DSC heating curve shows a broad endothermic peak located at ca. $475^{\circ} \mathrm{C}$. This feature is absent on the cooling curve and this observation points that the transition is irreversible. The endothermic peak was also absent when the sample was conducted to the repeated heating/cooling cycles (data now shown). The transition observed on the DSC curve can be ascribed to the decomposition of austenite. Wen et al. studied the thermal stability of retained austenite in low carbon steel and found that the retained austenite decomposes at $495-497^{\circ} \mathrm{C}$ [28]. The observed phase transition temperature is lower and is plausibly a consequence of the grain size. It has been demonstrated that the phase transitions in nanoparticles occurs at lower temperature in comparison to bulk materials [29]. The magnetic studied support the hypothesis that that the decomposition of austenite was successful. Fig. 10 shows the hysteresis loops for the non-annealed and annealed CEINs obtained with the addition of Ti. The saturation magnetization substantially increases after heat treatment, i.e. from 73 to $97 \mathrm{emu} / \mathrm{g}$. The saturation magnetization of the annealed sample is still below the maximum achievable value. The Fe content in this sample is $59.5 \mathrm{wt} . \%$ and this should reflect the saturation magnetization of $133 \mathrm{emu} / \mathrm{g}$ (224 emu/g.0.595$133 \mathrm{emu} / \mathrm{g})$. The experimental value $(97 \mathrm{emu} / \mathrm{g}$ ) is only $73 \%$ of the predicted magnetization. This finding implies that: (i) annealed sample contains some amounts of retained austenite, and (ii) the annealed materials contains iron carbide which was not transformed to ferrite and graphite. In fact, iron carbide which has lower saturation magnetization (ca. $165 \mathrm{emu} / \mathrm{g}$ ) in comparison to pure bcc Fe phase and its presence may reduce the observable

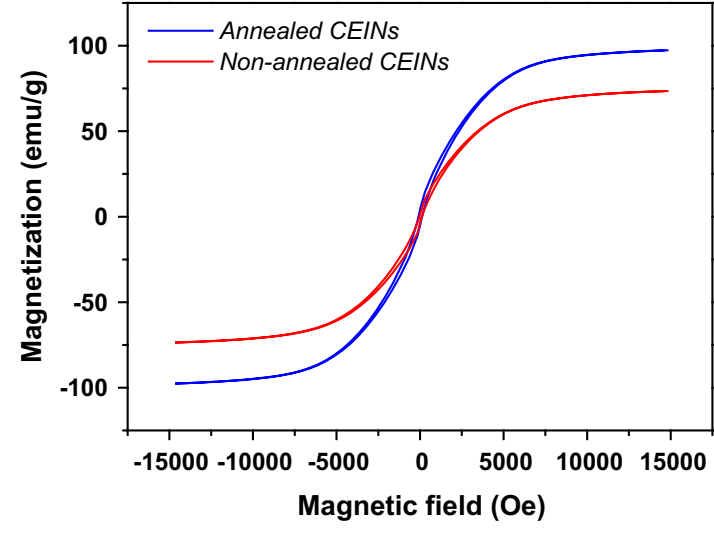

Fig. 10. Magnetic hysteresis loops for non-annelad and annelad purified CEINs obtained with addition of titanium.

saturation magnetization. The exact solution of this problem could be derived from detailed studies by Mössbauer spectroscopy.

\section{Conclusions}

The aim of this work was to verify whether is it possible to minimize the amount of undesired paramagnetic austenite phase in carbon-encapsulated iron nanoparticles synthesized via thermal plasma jet route. The hypothesis included the incorporation of eight elements which are widely used in steel metallurgy as ferrite stabilizing elements. For the sake of comparison Co (austenite stabilizing additive) was also investigated. The inclusion of all studied additives influenced the major operational parameters (purification rate, product formation rate) as well as the process selectivity, phase and chemical composition, graphitization degree and magnetic properties. All of the additives reduced the mean diameter of carbon-encapsulated iron nanoparticles. The largest reduction (more than twofold) was found for $\mathrm{Al}$ and Mo. The inclusion of $\mathrm{Al}$, $\mathrm{Ti}, \mathrm{Cr}$ and $\mathrm{V}$ changed the process selectivity. The products, beside carbon encapsulates, contained some amount of few layer graphene. The presence of the additives strongly influenced the phase composition. In most cases the products, beside Fe-bearing phases, contained respective carbides. The products had soft ferromagnetic behavior with the corresponding coercive field between 40 and 173 Oe. The remnant magnetization varied between $1.5 \%$ and $6.3 \%$ of the saturation magnetization. The inclusion of any of the studied ferrite stabilizing elements drastically worsened the magnetic performance. This was manifested by the decreased saturation magnetization, which resulted from an increased content of the paramagnetic austenite phase. It has been also demonstrated that non-sophisticated annealing of carbon-encapsulated iron nanoparticles under inter atmosphere significantly improves their saturation magnetization.

\section{Acknowledgements}

This work was supported by the National Centre for Research and Development (Poland) through the Project LIDER 527/L-4/ 2012 "New mobile nanocomposites of high corrosion resistance for removal of aromatic compounds and heavy metal ions".

\section{Appendix A. Supplementary material}

Supplementary data associated with this article can be found, in the online version, at http://dx.doi.org/10.1016/j.jallcom.2014.09. 044. 


\section{References}

[1] B.R. Elliott, J.J. Host, V.P. Dravid, M.H. Teng, J.H. Hwang, J. Mater. Res. 12 (1997) 3328-3344.

[2] I.K. Herrmann, R.N. Grass, W.J. Stark, Nanomedicine 4 (2009) 787-798.

[3] M. Bystrzejewski, S. Cudziło, A. Huczko, H. Lange, J. Alloys Comp. 423 (2006) 74-76.

[4] P. Lukanov, V.K. Anuganti, Y. Krupskaya, A.M. Galibert, B. Soula, C. Tilmaciu, A.H. Velders, R. Klingeler, B. Büchner, E. Flahaut, Adv. Funct. Mater. 21 (2011) 3583-3588.

[5] M. Bystrzejewski, R. Klingeler, T. Gemming, B. Bucher, M.H. Rummeli, Nanotechnology 22 (2011) 315606.

[6] J.H. Park, J. Jung, P. Subramaniam, B.P. Shah, C. Kim, J.K. Lee, J.H. Cho, C. Lee, K.B. Lee, Small 7 (2011) 1647-1652.

[7] M. Bystrzejewski, J. Sol. State Chem. 184 (2011) 1492-1498.

[8] M. Bystrzejewski, Z. Karoly, J. Szepvolgyi, W. Kaszuwara, A. Huczko, H. Lange, Carbon 47 (2009) 2040-2048.

[9] M. Bystrzejewski, O. Łabędź, W. Kaszuwara, A. Huczko, H. Lange, Powder Technol. 246 (2013) 7-15.

[10] I.K. Herrmann, R.N. Grass, D. Mazunin, W.J. Stark, Chem. Mater. 21 (2009) 3275-3281.

[11] H. Zhang, J. Phys. Chem. Sol. 60 (1999) 1845-1847.

[12] M. Bystrzejewski, A. Grabias, J. Borysiuk, A. Huczko, H. Lange, J. Appl. Phys. 104 (2008) 54307.

[13] O. Łabędź, A. Grabias, W. Kaszuwara, M. Bystrzejewski, J. Alloys Comp. 603 (2014) 230-238.
[14] K.A. Foglein, I. Babievskaya, P.T. Szabo, J. Szepvolgyi, Plasma Chem. Plasma Process. 23 (2003) 233-243.

[15] M. Bystrzejewski, Z. Karoly, J. Szepvolgyi, A. Huczko, H. Lange, Mater. Res. Bull. 46 (2011) 2408-2417.

[16] Y. Han, J. Shi, L. Xu, W.Q. Cao, H. Dong, Mater. Des. 34 (2012) 427-434

[17] J. Giddings, G.M. Leak, R.B. Nicholson, Mater. Sci. Technol. 8 (1974) 349-352.

[18] Y. Saito, T. Matsumoto, K. Nishikubo, J. Cryst. Growth 172 (1997) 163-170.

[19] T. Oku, T. Hirata, N. Motegi, R. Hatakeyama, N. Sato, T. Mieno, N.Y. Sato, H. Mase, M. Niwano, N. Miyamoto, J. Mater. Res. 15 (2000) 2182-2186.

[20] S. Bandow, Y. Saito, Jpn. J. Appl. Phys. 32 (1993) L1677-L1680.

[21] E.B. Barros, N.S. Demir, A.G. Souza Filho, J.M. Filho, A. Jorio, G. Dresselhaus, M.S. Dresselhaus, Phys. Rev. B 71 (2005) 165422.

[22] L.G. Cancado, K. Takai, T. Enoki, M. Endo, Y.A. Kim, H. Mizusaki, N.L. Speciali, A. Jorio, M.A. Pimenta, Carbon 46 (2008) 272-275.

[23] M.S. Dresselhaus, A. Jorio, R. Saito, Annu. Rev. Condens. Matter Phys. 1 (2010) 89-108.

[24] A. Oya, J. Mater. Sci. 17 (1982) 309-322.

[25] M. Bystrzejewski, A. Huczko, H. Lange, S. Cudziło, W. Kiciński, Diamond Relat. Mater. 16 (2007) 225-228.

[26] Z. Wang, P. Xiao, N. He, Carbon 44 (2006) 3277-3284.

[27] J.A. da Cruz, D.B. Santos, J. Mat. Res. Technol. 2 (2013) 93-99.

[28] W. Shi, L. Li, B.C. De Cooman, P. Wollants, C. Yang, J. Iron Steel Res. Int. 15 (2008) 61-64.

[29] P. Ding, J. Ma, H. Cao, Y. Liu, L. Wang, J. Li, Mat. Sci. Eng. B 178 (2013) 930-936. 